
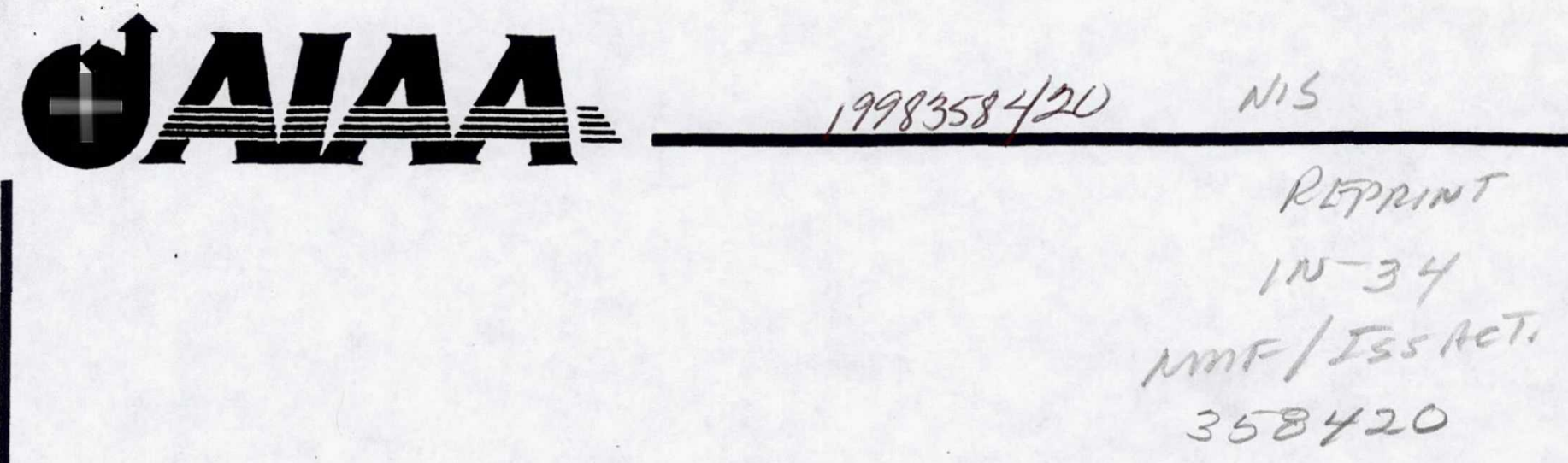

\title{
AIAA-98-3276 \\ Reduction of Jet Penetration in a Cross-flow by using Tabs
}

K. B. M. Q. Zaman

NASA Lewis Research Center

Cleveland, OH 44135

\section{4th AIAA/ASME/SAE/ASEE Joint Propulsion Conference \& Exhibit July 13-15, 1998 / Cleveland, $\mathrm{OH}$}




\title{
Reduction of Jet Penetration in a Cross-flow by using Tabs
}

\author{
by \\ K. B. M. Q. Zaman \\ NASA Lewis Research Center \\ Cleveland, $\mathrm{OH} 44135$
}

\begin{abstract}
A tab placed suitably on a nozzle that produces a jet in a cross-flow can reduce the penetration of the jet. This effect, achieved when the tab is placed on the windward side of the nozzle relative to the cross flow, may be of interest in film cooling applications. Wind tunnel experiments are carried out, in the momentum ratio $(J)$ range of $10-90$, to investigate the tab geometry that would maximize this effect. The preliminary results show that a 'delta tab' having a base width approximately fifty percent of the nozzle diameter may be considered optimum. With a given tab size, the effect is more pronounced at higher $J$. Reduction in jet penetration by as much as $40 \%$ is observed. Comparable reduction in jet penetration is also obtained when a triangular shaped tab is placed flush with the tunnel wall or with its apex tilted down into the jet nozzle (the 'delta tab' being the configuration in which the apex is tilted up). However, the delta tab involves the least flow blockage and pressure loss. Relative to the baseline case, the lateral spreading of the jet is found to be more with the delta tab but less with other orientations of the tab.
\end{abstract}

\section{Introduction}

In an effort to increase mixing and penetration of a jet in a cross flow, the effect of vortex generators in the form of tabs was investigated in the work reported in Refs. 1 and 2. The experiments involved a jet discharging normally from the floor of the wind tunnel test section, and various tab configurations were tried. The tabs, for the intended purpose, were found ineffective in both studies. The ineffectiveness was qualitatively explained in Ref. 2 to be due to the characteristic static pressure distribution near the exit of the jet nozzle. Specifically, the location (leeward side of the nozzle relative to the cross flow) where the tab would be expected to generate a vortex pair with the same sense of rotation as that of the 'bound vortex pair', was characterized by a drop in the static pressure. The low pressure occurred apparently due to streamline curvature. The resultant 'pressure valley', however, canceled the 'pressure hill' generated by the tab. This caused the ineffectiveness because the primary source of streamwise vortcity in the flow over a tab is the 'pressure hill' (Ref. 3).

The results of Ref. 2, on the other hand, showed that a significant opposite effect could be achieved when the tab was placed, relative to the cross stream, on the windward side of the nozzle. The vortex pair produced by the tab then had a cancellation effect on the strength of the bound vortex pair. The reduced strength led to a reduced mutual induction of the bound vortex pair. This led to a substantial reduction in the penetration of the jet.

The latter effect is of interest in film cooling applications where it is desirable that the jet with the coolant flow hugs the surface and does not penetrate and get mixed with the hot cross- 
stream for as long as possible (Ref. 4). This potential application inspired further wind tunnel experiments. Tests were conducted with a simple flow configuration, described further in the following, to optimize the tab geometry that would maximize the reduction in the jet penetration. Unfortunately, time and other constraints did not permit a complete and thorough investigation. The present paper is a status report of the ongoing effort.

\section{Experimental Procedure}

The experiment was conducted in a low speed wind tunnel with a $76 \mathrm{~cm} \times 51 \mathrm{~cm}$ test section. The flow arrangement is schematically shown in Fig. 1. A $2.23 \mathrm{~cm}$ diameter $(D)$ jet discharged normally from the floor of the test section. The air flow for the jet was routed through a small plenum chamber fitted with flow conditioning units. The velocity profiles at the nozzle exit, with no flow in the wind tunnel $\left(U_{T}=0\right)$, were found to be uniform (top-hat). The average jet speed $\left(V_{j}\right)$, with or without the tunnel flow on, was determined from measurement of the mass flow rate via an orifice-meter fitted to the supply line.

Triangular shaped tabs were placed on the windward side of the nozzle. The tab placement and its geometry is shown in the inset at the top of Fig. 1. The tab is triangular in shape with an apex angle of $90^{\circ}$. The base of the triangle is placed at the edge of the nozzle exit, with the plane of the triangle making an angle of $\theta$ as shown. Note that a part of the tab surface, in the sector between the nozzle's edge and the bend in the tab, is perpendicular to the jet stream. The 'delta tab' refers to the configuration when $\theta=$ $45^{\circ}$. It should be noted that the tabs were hand cut and installed under visual inspection. Thus, the dimensions were not precise. The tab size, orientation $\theta$, and the momentum flux ratio $J$ (= $\left.\left(\rho_{j} V_{j} / \rho_{T} U_{T}\right)^{2}\right)$ were varied in the investigation. The flow field surveys were conducted with a single hot-wire under automated computer con-

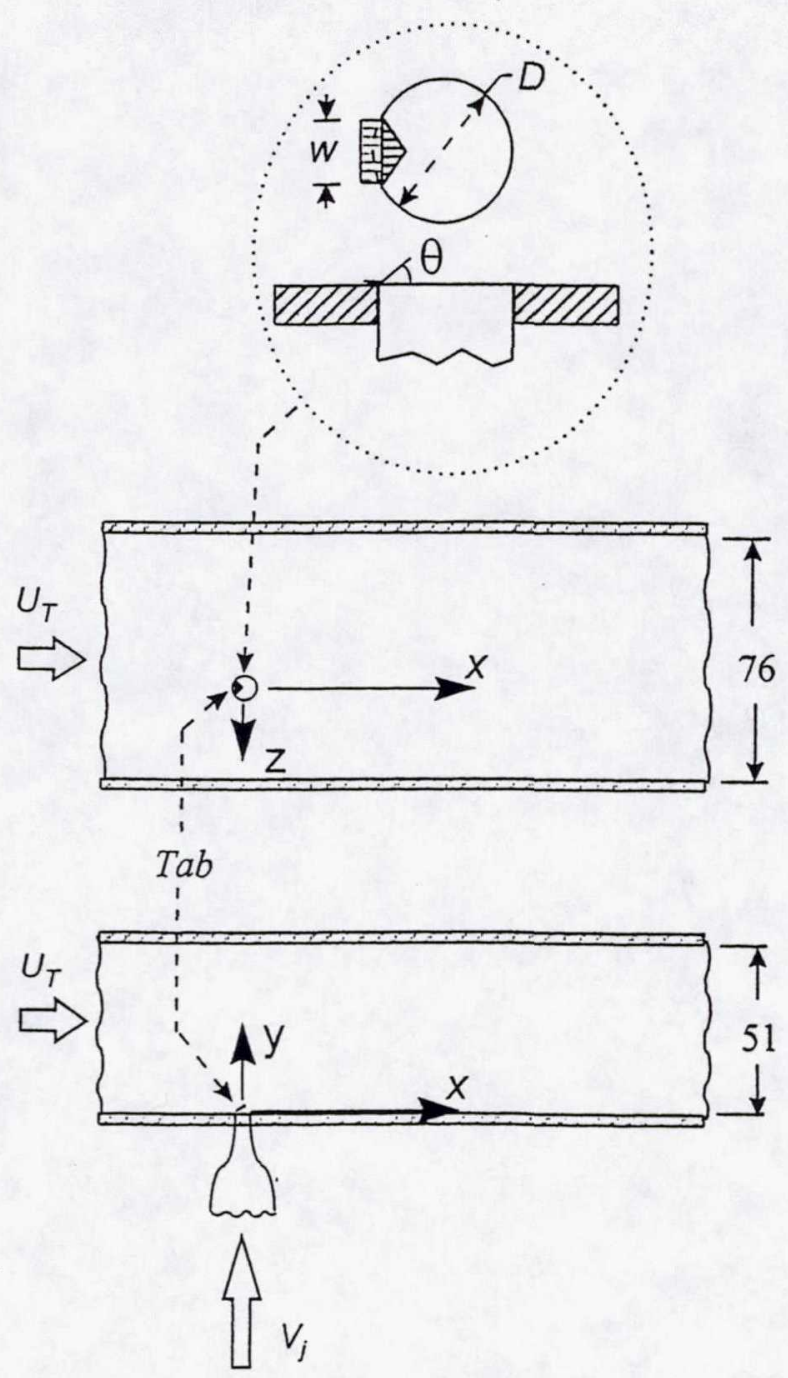

Fig. 1 Schematic of wind tunnel test section and jet; dimensions in $\mathrm{cm}$. Inset at top shows tab placement.

trol. For further details of the experimental procedure the reader may consult Ref. 2 .

\section{Results}

Figure 2, reproduced from Ref. 2, shows the mean velocity contours measured on the $y-z$ plane at $x / D=4$; the coordinate system has been shown in Fig. 1. The two sets of data are without and with a delta-tab located on the windward side. An inspection reveals the 'kidney-shaped' distribution of the high momentum fluid, over a core of low momentum fluid, in both cases. A reduction in the penetration of the high momen- 


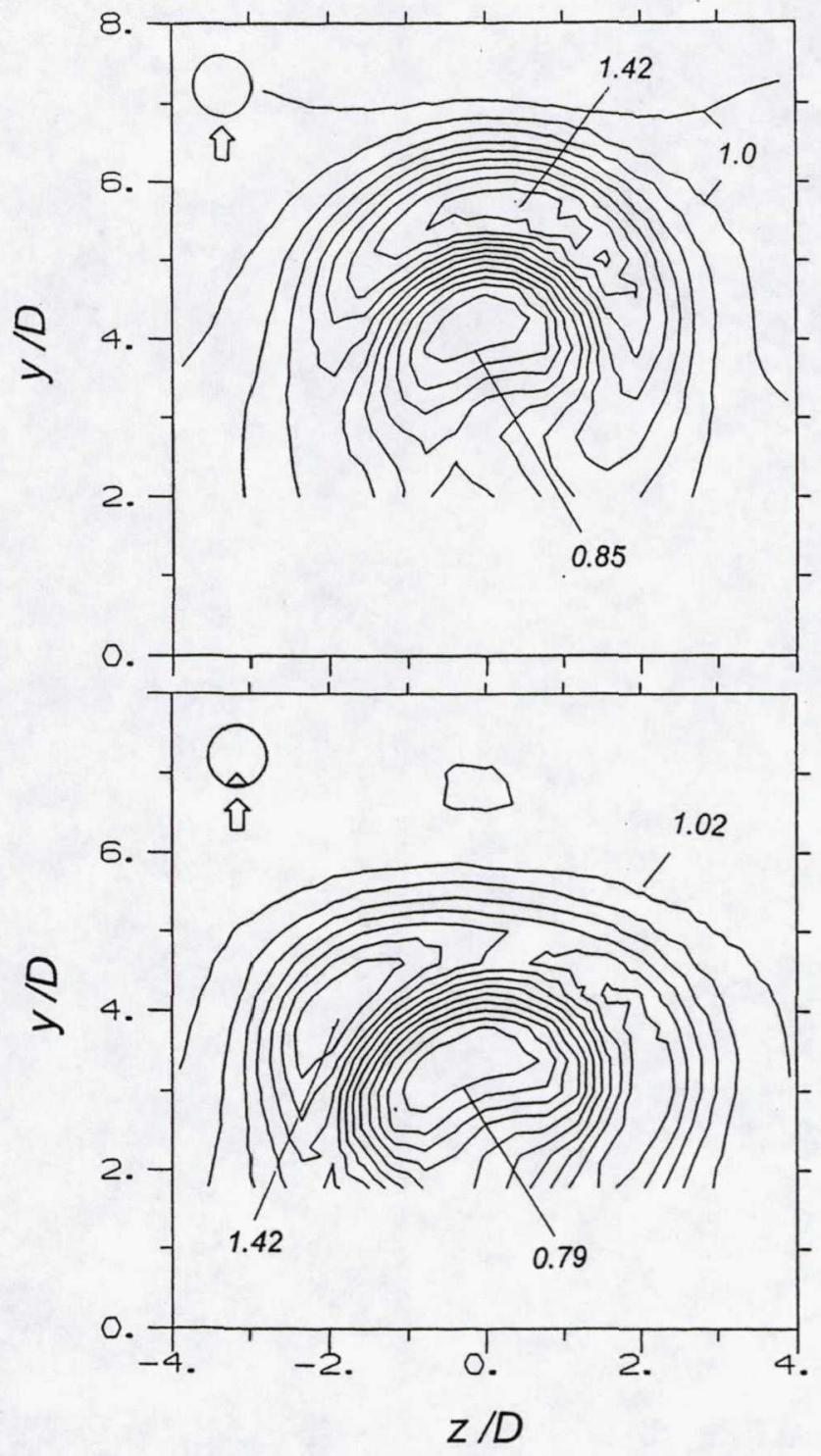

Fig. 2 Mean velocity $\left(U / U_{T}\right)$ distribution at $x / D=$ 4 for $J_{S E T}=21$; no-tab case on top and tab case $\left(w / D=0.35, \theta=45^{\circ}\right)$ at bottom. Contour intervals are 0.052 (top) and 0.057 (bottom).

tum fluid under the influence of the tab should also be obvious. At the center plane $(z=0)$, the location of the maximum velocity region has shifted from about $5.5 \mathrm{D}$ to about $4.5 \mathrm{D}$. In the caption of this figure, the notation $J_{\text {set }}$ represents an approximate value of $J$ without taking into consideration the flow blockage by the tab. This was set by calculating $V_{j}$ from the measured mass flow rate assuming no blockage by the tab. For a relatively small delta tab as in the case of Fig. 2

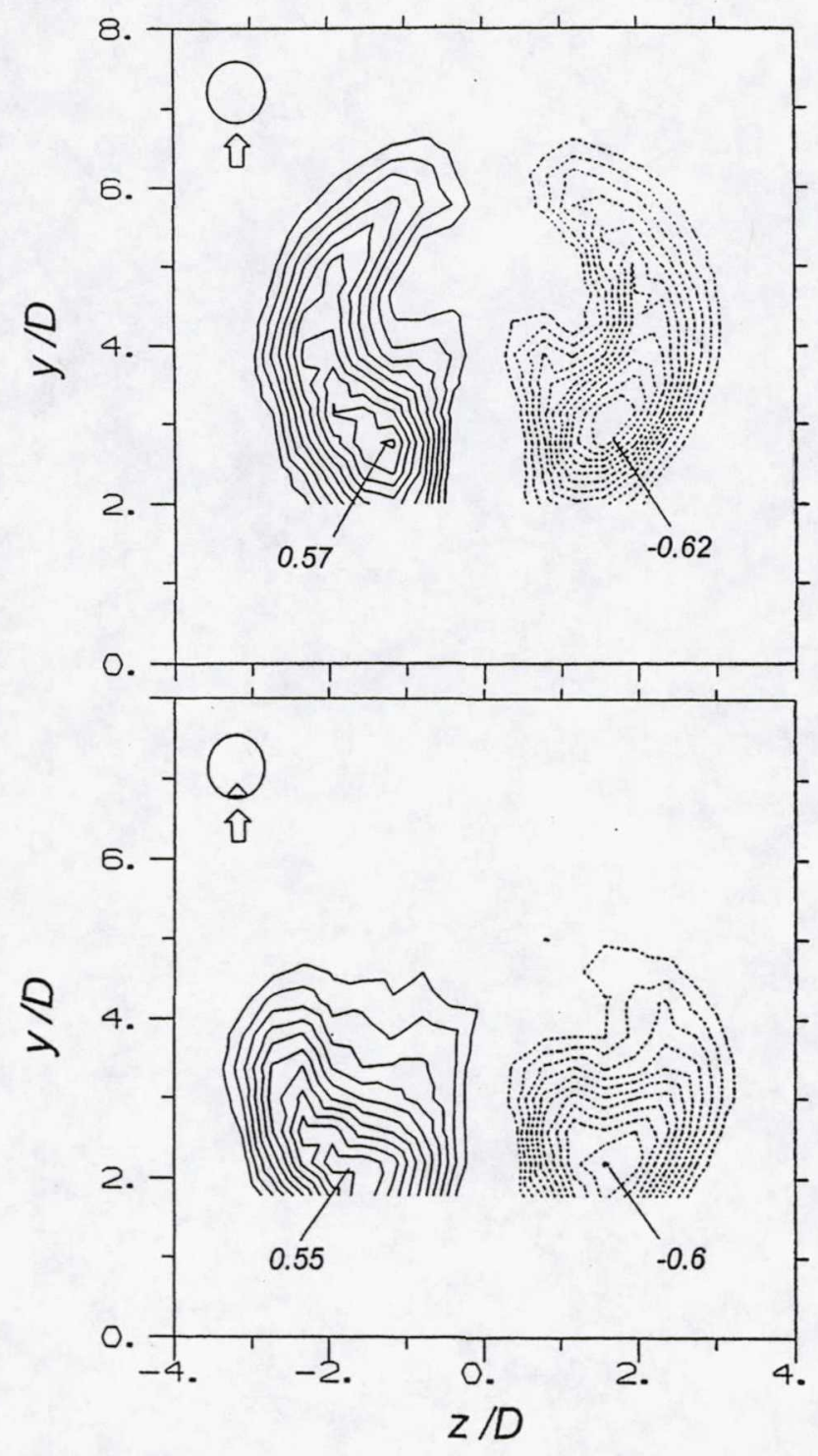

Fig. 3 Streamwise vorticity $\left(\omega_{X} D / U_{T}\right)$ distribution corresponding to the data of Fig. 2; contour intervals are 0.052 (top) and 0.05 (bottom). $(w / D=0.35) J_{\text {set }}$ is close to the actual value of $J$. But with larger size and different orientation of the tab the flow blockage is large; this would result in a large difference between $J_{\text {set }}$ and $J$. This blockage effect is considered duly in the following.

Figure 3 shows the time-averaged streamwise vorticity distribution corresponding to the data of Fig. 2. The upper figure shows the counter-rotating 'bound vortex pair' that charac- 


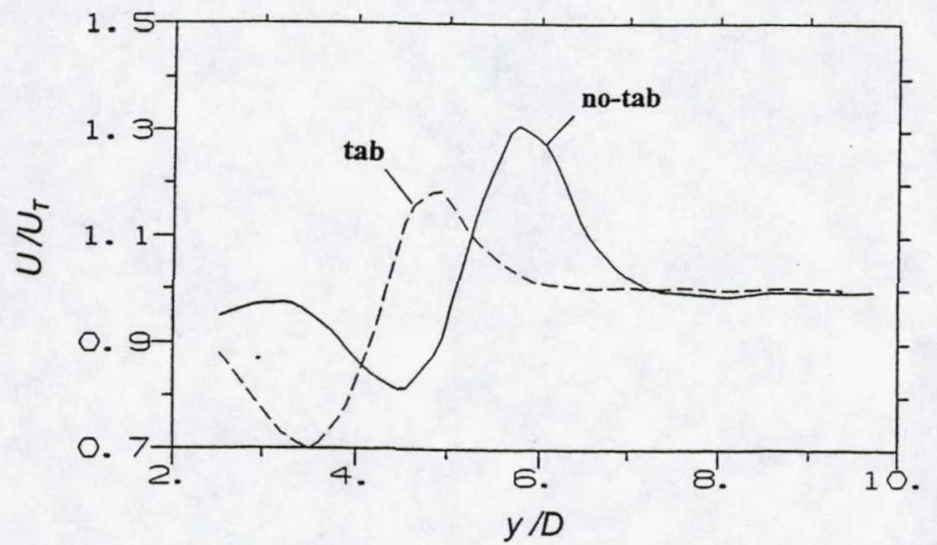

Fig. 4 Mean velocity profile with and without tab $(w / D=0.57)$ at $x / D=6 ; J_{S E T}=17$.

terizes all jets in a cross-flow. The sense of this vortex pair (solid and dashed contours represent counter clockwise and clockwise rotation, respectively) is such that low momentum fluid from near the tunnel floor $(y=0)$ is pulled up. The mutual induction of the vortex pair also causes the pair to lift upwards, causing the upward penetration of the jet. The tab has clearly weakened the bound vortex pair, as evident from the distribution in the lower figure. When placed on the windward side, the tab produces a vortex pair with a sense opposite to that of the bound vortex pair. This results in a cancellation effect. As discussed further in Ref. 2, this is thought to be the primary reason for the lesser jet penetration caused by the tab. In the following, this effect is explored further. Only measurements on the $z=0$ plane, deemed sufficient for this exploration, are reported from here on.

In order to clarify certain terminology to be used in the following, examples of mean velocity profiles with and without the tab, measured at $x / D=6(z=0)$, are shown in Fig. 4. The shift of the peak in the $U(y)$ profile towards the tunnel floor caused by the tab can be clearly seen. Note that the lower speed flow $\left(U / U_{T}<1\right)$ underneath the peak velocity region is due to the ingestion of the boundary layer fluid. The location of the peak in the $U(y)$ profile, designated as 4 $y_{\text {max }}$, is used as a measure of the jet penetration. The locus of $y_{\max }$ with varying $x$ is referred to as the 'jet trajectory'. The jet trajectory is obtained by hot-wire surveys for the various tab configurations.

First, the jet trajectory for the no-tab case is shown in Fig. 5 for different momentum flux ratio, $J$. Curves, $y=J^{0.45} x^{0.33}$, best fitting the entire set of data, are also shown. Note that the exponents in the equation are slightly different from those suggested in earlier work (Ref. 5). In the following, the measured trajectories for the tab cases are compared with the corresponding no-tab case represented by this correlation equation.

Figure 6 shows the effect of a delta tab on the jet trajectory while the size of the tab is varied. A decreasing jet penetration with increasing tab size is apparent. These data are for $J_{\text {set }}=35.5$. As stated before, with increasing blockage the effective diameter $\left(D_{e}\right)$ becomes smaller than $D$, and the actual value of $J$ becomes substantially

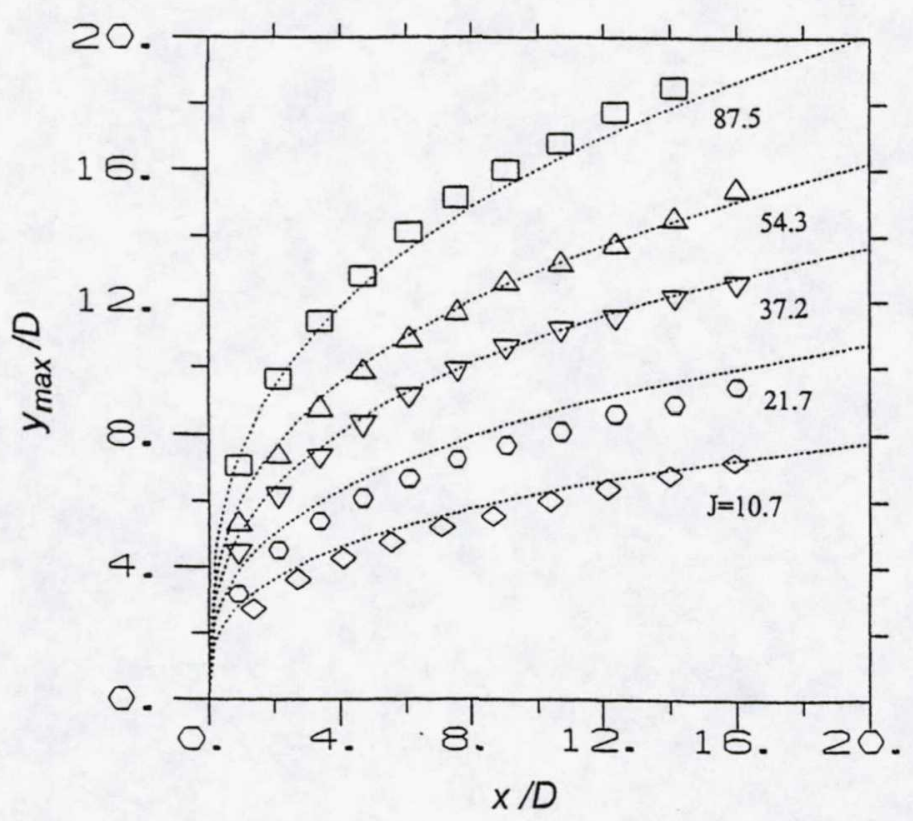

Fig. 5 'Jet trajectory', $y_{\max }$ versus $x$, for the no-tab case; dotted lines, $y=J^{0.45} x^{0.33}$. 


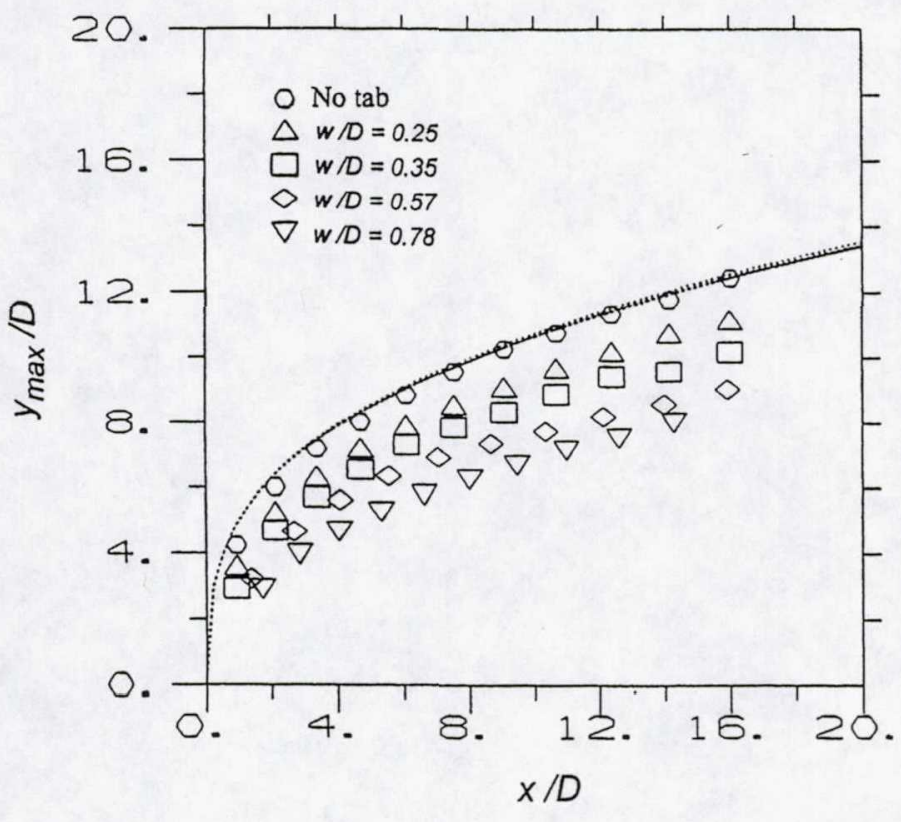

Fig. 6 'Jet trajectory' for the effect of a tab with indicated size $\left(\theta=45^{\circ}\right) ; J_{S E T}=35.5$.

larger than $J_{\text {ser }}$. In order to assess the effect of the tab properly, $y_{\max }$ and $\mathrm{x}$ need to be nondimensionalized by $D_{e}$ and the actual $J$ needs to be set properly. These are done for the following data.

First, samples of data used to determine the equivalent diameter and the flow blockage are shown in Fig. 7. For a tab with fixed size $(w / D=$ $0.57)$ and three different orientations $\left(\theta=45^{\circ}, 0^{\circ}\right.$ and $\left.-45^{\circ}\right)$, the mass flow rate $(m)$ is shown as a function of the jet plenum chamber pressure. The upper set of data is for zero tunnel speed while the lower set is for a fixed tunnel speed as indicated. The mass flow rate, for a given pressure, is lower for the tab cases compared to the no-tab case, as expected. From these data, a fluid dynamic blockage at a given pressure is calculated as, $\left(m_{\text {notab }}\right.$ $\left.m_{\text {tab }}\right) / m_{\text {notab }}$. Assuming incompressible flow, the equivalent diameter is determined as, $D_{e} / D$ $=\mathrm{SQRT}\left(m_{t a b} / m_{\text {notab }}\right)$. These values are calculated at three values of the pressure $(0.28,0.55$ and $0.83 \mathrm{kPa}$ ). The value of $D_{e} / D$ at the three pressures for a given tab case varied only slightly, within $2 \%$. Averages of the three values were

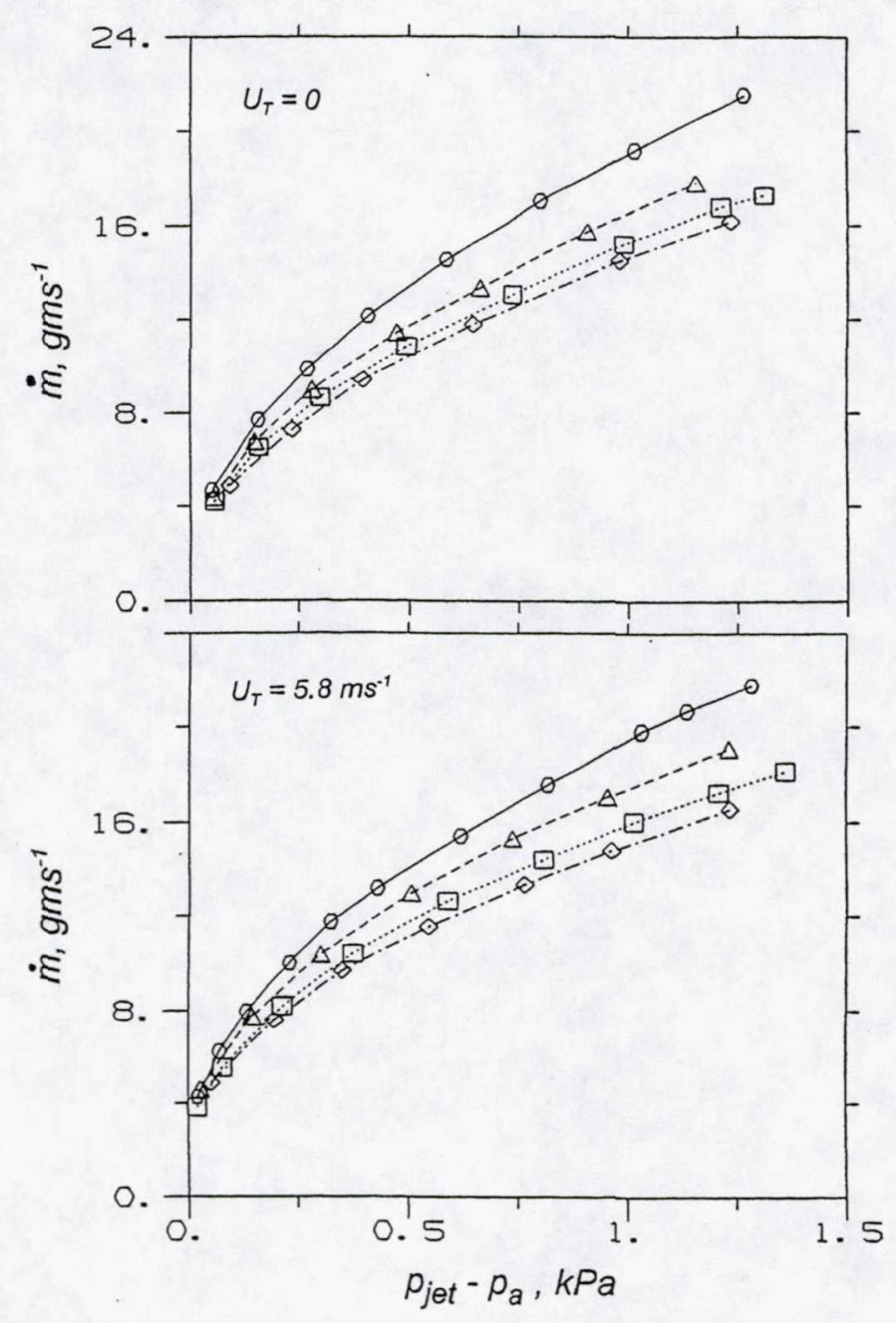

Fig. 7 Variation of mass flow rate with jet plenum chamber pressure for indicated tunnel speeds.

O, no-tab; $\Delta, \theta=45^{\circ} ; \square, \theta=0^{\circ} ; \diamond, \theta=-45^{\circ}$; $w / D=0.57$ for all tabs.

taken to represent $D_{e}$ for the given tab case. The values, in turn, were found to differ only slightly with or without the tunnel flow on. The latter observation should be apparent from an inspection of the two sets of data in Fig. 7. The fluid dynamic blockage and $D / D$ for all the tab cases are listed in Table 1.

However, since the tabs were positioned under visual inspection, every time a tab was reinstalled, $D_{e} / D$ and the blockage did not reproduce exactly. Table 1 shows typical values. The scatter in $D_{e} / D$ from setup to setup for the same tab configuration was about $5 \%$. In order to alleviate the 


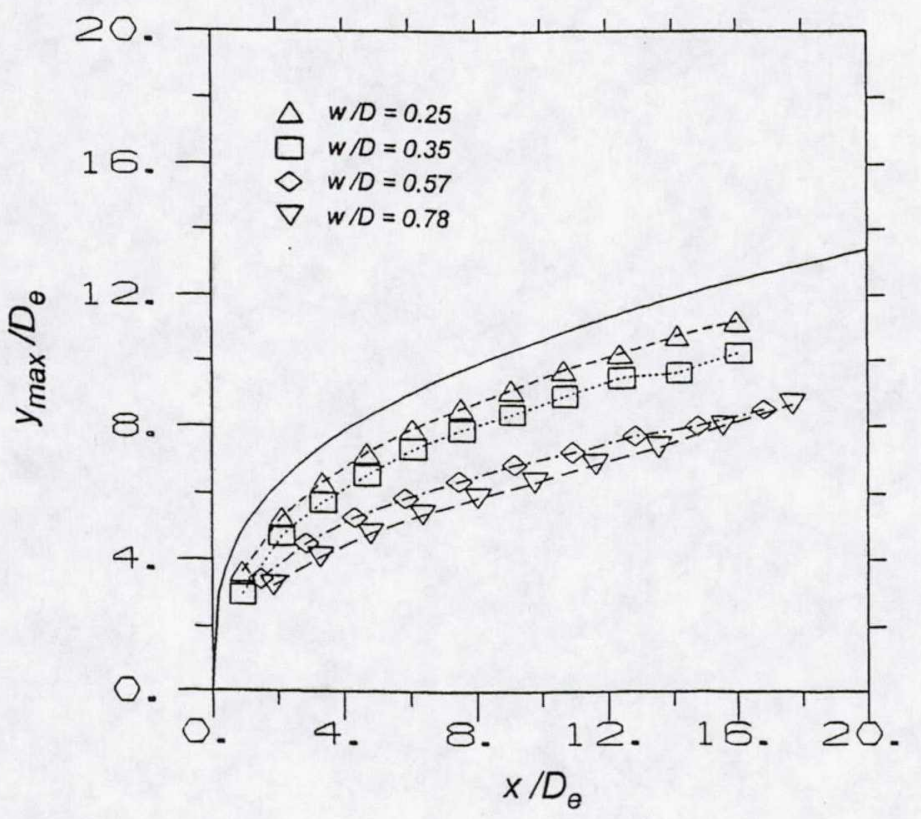

Fig. 8 Effect of tab $\left(\theta=45^{\circ}\right)$ with varying size $(w / D)$ on the 'jet trajectory'; $J=36$.

effect of this uncertainty, $D / D$ and blockage were always measured first before acquiring other data. It can be seen from Table 1 that the blockage is large with the large tabs and for $\theta=-45^{\circ}$. The corresponding values of $D / D$ are thus considerably lower than unity. Also, recalling that $J_{\text {set }}$ is obtained from the measured mass flow rate with the assumption of no flow blockage, it follows that, $J / J_{\text {set }}=\left(D_{e} / D\right)^{4}$. This relationship was used to set up a particular value of $J$ during the experiments.

Data similar to those in Fig. 6 were obtained for a fixed value of $J$, following the considerations discussed above, and are shown in Fig. 8. The solid line in this figure represents the correlation equation for the baseline (no-tab) case as discussed before. It is clear that a trend similar to that seen in Fig. 6 still holds. Increasing the tab size resulted in a reduced penetration of the jet. However, increasing the size from $w / D=0.57$ to $w / D=0.78$ yielded a 'diminishing return'. Only a slight reduction in the penetration is achieved

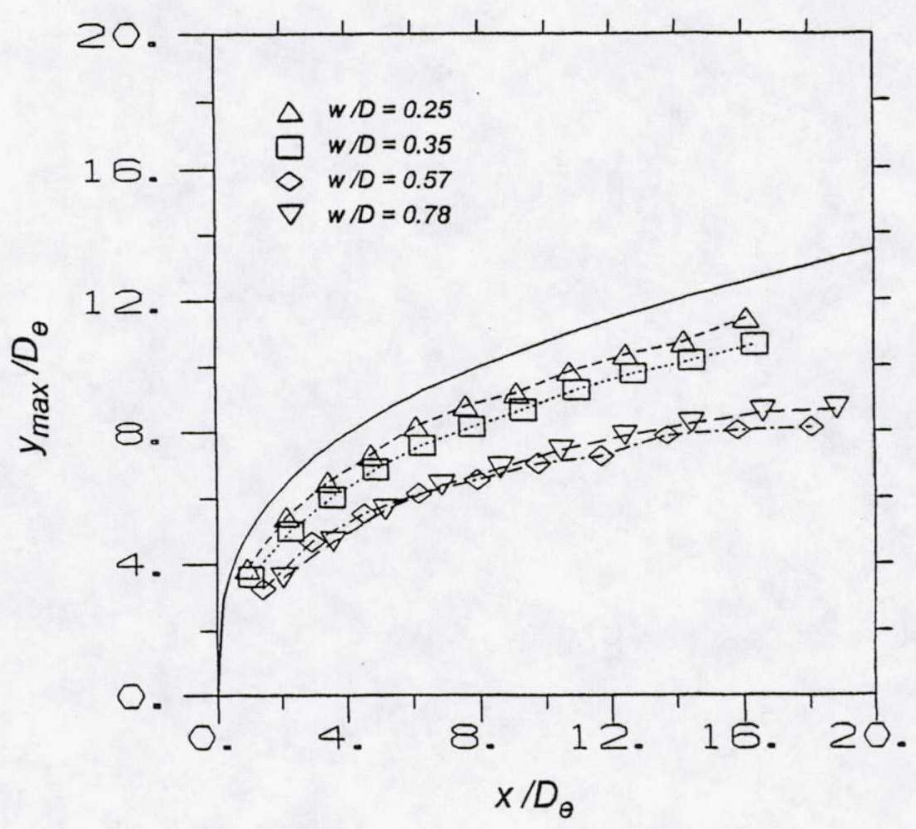

Fig. 9 Effect of $\operatorname{tab}\left(\theta=-45^{\circ}\right)$ with varying size $(w / D)$ on the 'jet trajectory'; $J=36$.

with the larger size tab. This effect is seen even more pronounced in Fig. 9 as discussed next.

Corresponding data for the same four tabs, as in Fig. 8, but placed inverted $\left(\theta=-45^{\circ}\right)$ are shown in Fig. 9. First, it is apparent that for a given tab size, orientation does not have a significant effect on the penetration and comparable reductions are achieved. Second, with the largest tab size, a reversal in the effect has actually taken place. Relative to the $w / D=0.57$ case, the $w / D=$ 0.78 case has resulted in an increase in the jet penetration in the downstream region.

For a delta tab with $w / D=0.57$ the jet trajectories for three different values of $J$ are shown in Fig. 10(a). The arrows show the deviation of the trajectories from the respective baseline (no-tab) curves. At the two higher values of $J$, about $35 \%$ reduction in $y_{\max }$ has been achieved throughout the $x$-range covered. At the lowest $J$, the reduction is not as much at about $20 \%$. Corresponding data for the effect of the tab with the same size but placed flush with the tunnel wall $(\theta$ 


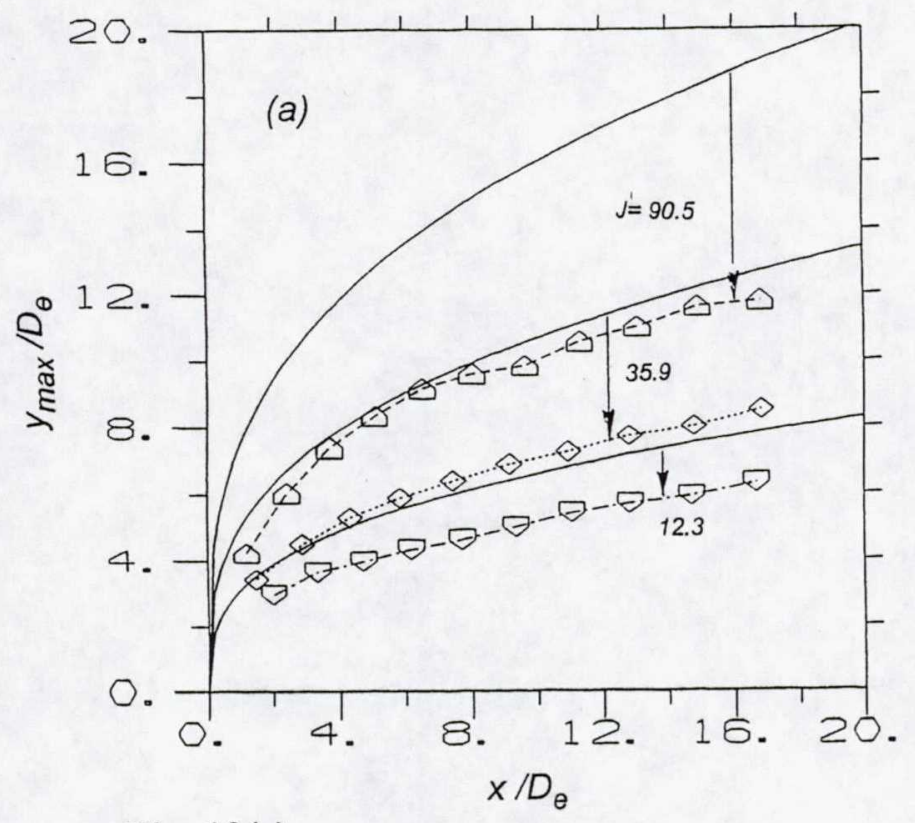

Fig. 10(a)

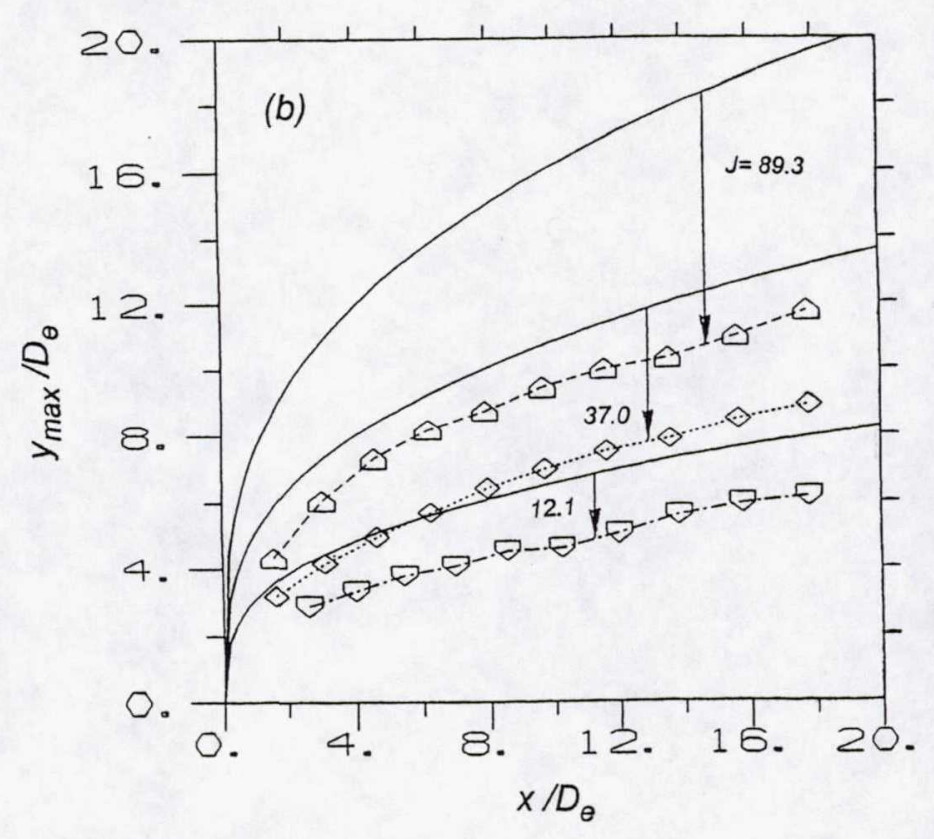

Fig. 10(b)

$=0^{\circ}$, see Fig. 1) are shown in Fig. 10(b), for the same three nominal values of $J$. Reductions comparable to those seen in Fig. 10(a) have occurred. Nominal reductions of $40 \%, 35 \%$ and $25 \%$ have been achieved at $J=89,37$ and 12 , respectively. Finally, corresponding data for tab with the orientation of $\theta=-45^{\circ}$ are shown in Fig. 10(c ). Again, reductions comparable to those seen in figures 10(a) and (b) are observed. However, the reduction occurring at the highest $J$ is somewhat less, about $25 \%$.

From the three sets of data in Fig. 10 it is apparent that the orientation of the tab had relatively little consequence as far as reduction in jet penetration is concerned. The $\theta=0^{\circ}$ (flush with tunnel wall) actually produced slightly better results. This configuration may be desirable in applications as the tab would not be directly exposed to hot cross flow. However, it should be apparent from table 1 that the flow blockage, and hence the pressure loss (Fig. 7) is significantly lower with the delta tab configuration. Thus, with this consideration in mind, the delta tab with a base width of $0.57 D$ is the optimum among the

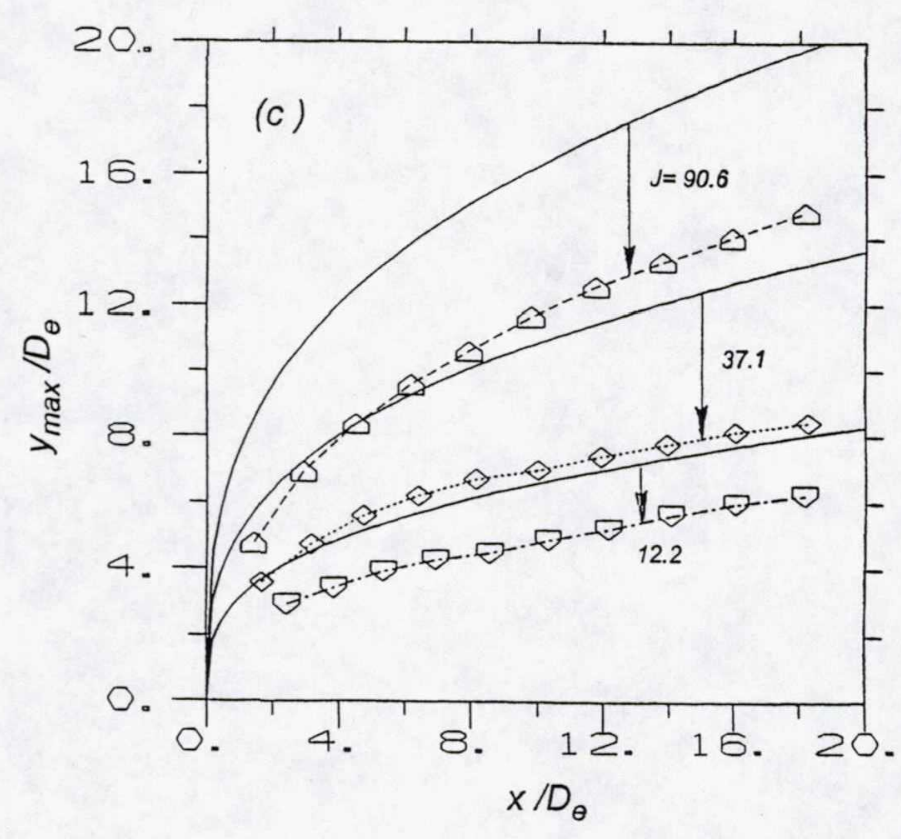

Fig. 10 Effect of a $w / D=0.57$ tab on 'jet trajectory' for indicated values of $J$; reduction in jet penetration from respective no-tab correlation curve is indicated by arrows. Tab inclination: (a) $\theta=45^{\circ}$, (b) $\theta=0^{\circ}$, (c ) $\theta=-45^{\circ}$. 
sizes and orientations tested so far. Finally, it should be mentioned that cursory surveys have indicated that the lateral spreading of the jet (in $z$ ) is significantly more with the delta tab. For example, at $x / D_{e}=14$, the width ( $\Delta z$ between peakto-peak in $U$ distribution) was found to be $67 \%$ of the baseline case for the $\theta=-45^{\circ}$ case but $113 \%$ for the $\theta=45^{\circ}$ (delta tab) case. These effects are being explored further with detailed flow field surveys.

\section{Concluding Remarks}

Results of a wind tunnel experiment are presented addressing jet penetration in a cross flow as affected by tabs. Relative to the cross flow, the tab was placed on the windward side of the nozzle. Tab size and orientation were varied. The jet trajectory was determined by measuring the locus of the maximum velocity point on the spanwise symmetry plane. A 'delta tab' generally reduced the jet penetration. For a given tab size, the reduction in jet penetration was more at higher values of the momentum flux ratio. The orientation of the tab, with a given size, did not affect the results significantly. With a given orientation, increasing the tab size resulted in a reduced jet penetration, until the base width of the tab was approximately $50 \%$ of the nozzle diameter. With flow blockage and pressure loss taken into account, a delta tab of this size may be considered as optimum. A full explanation for the observed effects remains incomplete. Detailed vorticity and pressure field measurements are being conducted to shed more light on the mechanisms of these effects.

\section{Acknowledgment}

The author would like to thank Mr. Frederick F. Simon for bringing to attention the relevance of the tab effect to film-cooling applications.

\section{References:}

(1) Liscinsky, D.S., True, B. and Holdeman, J.D., "Effects of initial conditions on a single jet in crossflow", AIAA Paper 95-2998, 31st Joint Prop Conf, San Diego, July 10-12, 1995.

(2) Zaman, K.B.M.Q. and Foss, J.K., "The effect of vortex generators on a jet in a cross-flow", Physics of Fluids, vol. 9 (1), pp. 106-114, 1997.

(3) Zaman K.B.M.Q., Reeder M.F., \& Samimy M., "Control of an axisymmetric jet using vortex generators", Physics of Fluids A, 6 (2), pp. 778-793, 1994.

(4) Hippensteele, S., "Film-cooling heat transfer measurements using liquid crystals", Proc. Coolant Flow Management Workshop, Dec. 1213, 1996, NASA CP 10195.

(5) Abramovich, G.N., "The theory of turbulent jets", The M.I.T. Press, 1963.

Table 1

Characteristics and flow blockage by the tabs

\begin{tabular}{|c|c|c|c|c|}
\hline $\begin{array}{l}\text { Base } \\
\text { width }\end{array}$ & $\begin{array}{l}\text { Inclina- } \\
\text { tion }\end{array}$ & $\begin{array}{l}\text { Geometric } \\
\text { blockage }\end{array}$ & $\begin{array}{l}\text { Measured } \\
\text { blockage }\end{array}$ & $D_{e} / D$ \\
\hline$w / D$ & $\theta$ & $(\%)$ & $(\%)$ & \\
\hline \multirow[t]{2}{*}{0.25} & $45^{\circ}$ & 1.8 & 0 & 1.00 \\
\hline & $-45^{\circ}$ & 1.8 & 1.5 & 0.992 \\
\hline \multirow[t]{2}{*}{0.35} & $45^{\circ}$ & 3.8 & 0.9 & 0.996 \\
\hline & $-45^{\circ}$ & 3.8 & 3.4 & 0.983 \\
\hline \multirow[t]{3}{*}{0.57} & $45^{\circ}$ & 11.7 & 2.4 & 0.988 \\
\hline & $0^{\circ}$ & 14.7 & 16.9 & 0.912 \\
\hline & $-45^{\circ}$ & 11.7 & 21.0 & 0.890 \\
\hline \multirow[t]{2}{*}{0.78} & $45^{\circ}$ & 26.7 & 14.6 & 0.92 \\
\hline & $-45^{\circ}$ & 26.7 & 42.9 & \\
\hline
\end{tabular}

